\title{
Patterns of phenoloxidase activity in insecticide resistant and susceptible mosquitoes differ between laboratory-selected and wild-caught individuals
}

Stéphane Cornet ${ }^{1,2,3^{*}}$, Sylvain Gandon ${ }^{2}$ and Ana Rivero ${ }^{1}$

\begin{abstract}
Background: Insecticide resistance has the potential to alter vector immune competence and consequently affect the transmission of diseases.

Methods: Using both laboratory isogenic strains and field-caught Culex pipiens mosquitoes, we investigated the effects of insecticide resistance on an important component of the mosquito immune system: the phenoloxidase (PO) activity. As infection risk varies dramatically with the age and sex of mosquitoes, allocation to PO immunity was quantified across different stages of the mosquito life cycle.

Results: Our results were consistent in showing that larvae have a higher PO activity than adults, females have a higher PO activity than males, and PO activity declines with adult age. We obtained, however, a marked discrepancy between laboratory and field-collected mosquitoes on the effect of insecticide resistance on PO activity. In the laboratory selected strains we found evidence of strong interactions between insecticide resistance and the age and sex of mosquitoes. In particular, 7 and 14 day old esterase-resistant adult females and acetylcholine-esterase resistant males had significantly higher PO activities than their susceptible counterparts. No such effects were, however, apparent in field-caught mosquitoes.

Conclusions: Combined, the field and laboratory-based approaches employed in this study provide a powerful test of the effect of insecticide resistance on PO-mediated immunity. The use of laboratory-selected insecticide-resistant strains is still the most widely used method to investigate the pleiotropic effects of insecticide resistance. Our results suggest that the outcome of these laboratory-selected mosquitoes must be interpreted with caution and, whenever possible, compared with mosquitoes captured from the field.
\end{abstract}

Keywords: Culex, Gender effect, Immune aging, Insecticide resistance, Phenoloxidase, Vector competence

\section{Background}

Mosquito-borne diseases, such as malaria, dengue and filariasis represent a major cause of illness and death worldwide. The extensive use of chemicals to control mosquito populations and reduce the burden of disease has led to the widespread emergence and spread of insecticide resistance [1]. Insecticide resistance counters

\footnotetext{
* Correspondence: stephan.cornet@gmail.com

'Maladies Infectieuses et Vecteurs: Ecologie, Génétique, Evolution et Contrôle (MIVEGEC), UMR CNRS 5290-IRD 224-UM1-UM2, Montpellier, France ${ }^{2}$ Centre d'Ecologie Fonctionnelle et Evolutive (CEFE), UMR CNRS 5175, Montpellier, France

Full list of author information is available at the end of the article
}

control methods by increasing vector population sizes above the critical thresholds required for disease management. Aside from its effect on vector numbers, however, insecticide resistance may also alter the quality of mosquitoes as vectors of disease [2,3]. In other words, insecticide resistant mosquitoes may be better (or worse) vectors of diseases than susceptible ones, a possibility which, if confirmed, could have drastic epidemiological and public health consequences [2].

There is a large body of work showing that the innate immune system is a key determinant of the vectorial capacity of mosquitoes: endogenous innate immune molecules

\section{Biomed Central}


hinder the development of malarial [4], filarial [5] and viral parasites [6]. In the last few years, a great deal of work has been undertaken to investigate the genetics and the molecular mechanisms underlying mosquito innate immune responses, on the one hand $[7,8]$, and insecticide resistance, on the other [9]. In spite of this, few studies have investigated whether insecticide resistance and immune response are connected [10]. However, insecticide resistance genes, or genes closely associated to them as a result of hitchhiking (see e.g. [11,12]) could have a pleiotropic effect on one of the many steps of the immune cascade, from the recognition of the parasite as foreign, to the transduction of the signal and the deployment of the killing mechanism. For example, insecticide resistance may interfere with immunity through resource-based trade-offs, as both insecticide resistance mechanisms [13] and the deployment of the immune machinery $[14,15]$ are energetically costly.

Here, we investigate whether insecticide resistance has a pleiotropic effect on the phenoloxidase activity of Culex pipiens mosquitoes. Culex mosquitoes are important vectors of diseases such as filariasis, West Nile, and Japanese encephalitis. $C x$. pipiens has also a well-deserved reputation for being one of the mosquito species where the molecular and genetic bases of insecticide resistance are best understood $[9,16]$. The phenoloxidase cascade is an important immune reaction of mosquitoes leading to the melanotic encapsulation and death of a variety of parasites [17-21]. In $C x$. pipiens it has been shown to be implicated in resistance against filarial parasites [22]. The melanotic encapsulation response results from the activation of a complex enzymatic cascade that ends up in the cleavage of inactive prophenoloxidases (proPO) into active phenoloxidases (PO), which initiate the production of melanin [23]. The activation of the cascade also generates toxic compounds that are efficient parasite killers [18,24]. The high diversification of the proPO genes revealed by comparative genomics has highlighted the importance of the melanization cascade in mosquito immunity $[25,26]$. In addition, the insect melanization response has been shown to be genetically correlated with other important components of the insect's immune system $[27,28]$, which has led to suggestions that the melanization cascade may be an indicator of the mosquito general immune competence.

The aim of the paper is to determine: (i) whether insecticide resistance affects mosquito PO activity and (ii) whether this effect varies between the larval and adult stages, and with the age and/or sex of the adults. We do the latter because allocation to immunity is a function of the level of parasite threat and of the costs associated to maintaining the trait [14], both of which vary widely throughout the life of a mosquito. Cx. pipiens larvae live in stagnant pools and are highly tolerant to organic and polluted water. As a result, larvae may be expected to have a stronger immune system than their adult counterparts (see e.g. [29]). Similarly, adult females may be expected to have a stronger immune system than males because blood feeding exposes them to a greater level and spectrum of parasites [30]. The immunity of both males and females is, however, expected to decline with age, as individuals shunt resources towards maximizing reproductive success before they die (immunosenescence, [31]). Immunosenescence is particularly relevant for disease vectors because, due to the long extrinsic incubation periods of many parasites, only relatively old individuals transmit diseases [32].

We carry out two separate experiments. In the first experiment (Experiment 1 ) we quantify PO activity in larvae, and in newly emerged (1-day), young (7-day) and old (14-day) male and female adults using three different isogenic $C x$. pipiens strains: a fully susceptible strain, a strain resistant to insecticides through the overproduction of esterases (metabolic resistance), and a resistant strain with an insensitive acetylcholinesterase (target site resistance). The construction of isogenic laboratory strains (whereby the resistance alleles are introgressed into a susceptible reference line by a repeated backcross and insecticide selection procedure, e.g. [33]) remains one of the most widely used methods for investigating the pleiotropic effects of insecticide resistance in mosquitoes. This procedure has several advantages in that it allows testing the effect of the insecticide resistance genes in a uniform genetic background, which may increase the chances of detecting an eventual pleiotropic effect of the insecticide resistance genes. It also has, however, several potential pitfalls: (i) the selection procedure requires that these mosquitoes are subjected to unnaturally high insecticide selective pressures [34], (ii) the selection procedure may inadvertently select for other mosquito traits, and (iii) the results may not be necessarily applicable to other genetic backgrounds, particularly if there are epistatic interactions between the insecticide resistant genes and other genes in the background [10].

To account for these potential sources of bias, we therefore repeated the same experiment using fieldcaught mosquitoes (Experiment 2). In the Montpellier region, repeated treatments of $C x$. pipiens larval sites with organophosphate insecticides (initiated 40 years ago) have resulted in the evolution of both esterase and acetylcholinesterase resistance. In this region, there is an insecticide-treated area (a $20 \mathrm{Km}$ band close to the sea), a non-treated area (further north), and an intermediate area where esterase-resistant and acetylcholinesteraseresistant mosquitoes coexist with susceptible ones [35]. Combined, the field and laboratory-based approaches we employ in this study provide a powerful test of the role of insecticide resistance on PO activity throughout the mosquito life cycle. 


\section{Methods}

\section{Experiment 1 - Isogenic strain mosquitoes}

We used three different isogenic strains of $C x$. pipiens (SLAB, SA4B4 and SR) that share the same (SLAB) genetic background and only differ by their genotype at the Ester (esterase over-production) or the ace-1 (insensitive acetylcholinesterase) loci: a fully susceptible strain (SLAB, alleles Ester ${ }^{0}$, ace- $1^{\mathrm{S}}$ ) and two strains resistant to insecticides, one through the overproduction of esterases A4 and B4 (SA4B4, alleles Ester ${ }^{4}$, ace- $1^{\mathrm{S}}$ ) and the other with an insensitive acetylcholinesterase but no overproduced esterases (SR, alleles Ester ${ }^{0}$, ace $-1^{\mathrm{R}}$ ) [16,33].

Synchronized Cx. pipiens L1 larvae cohorts from the three insecticide resistant strains were obtained from the Weill's lab at the Institut des Sciences de l'Evolution de Montpellier (France) and maintained under our standard insectary conditions (12 h:12 h photoperiod, $25 \pm 1^{\circ} \mathrm{C}$, $75 \pm 5 \% \mathrm{RH})$. Larvae were reared in plastic trays $(25 \times$ $35 \times 7 \mathrm{~cm}, 4$ trays per strain) filled with $1 \mathrm{~L}$ of source water (Carrefour) at an initial density of 300. Larvae were fed with fish food (Microfood, Tretramin ${ }^{\odot}$ ) and water was renewed each time food was provided. Each tray was placed inside a mesh cage $(27 \times 40 \times 35 \mathrm{~cm})$ for adult emergence (around day 10). Adults were provided with an ad libitum 10\% sugar solution for the duration of the experiment.

\section{Experiment 2- Field-caught mosquitoes}

Wild Cx. pipiens mosquito eggs were collected in JuneJuly 2011 from a population (Triadou, $20 \mathrm{~km}$ north Montpellier, France) where the insecticide susceptible $\left(\right.$ Ester $^{0}$, ace $\left.^{-1}{ }^{\mathrm{S}}\right)$ and resistant $\left(\right.$ Ester $^{4}$, ace $\left.^{\mathrm{R}} \mathrm{R}^{\mathrm{T}}\right)$ alleles are found in sympatry [36]. After hatching, 4 trays of 400 L1 larvae each were set up. Mosquitoes were reared and maintained under identical laboratory conditions as described above.

\section{Experimental procedure}

Both experiments were performed in an identical manner. The immune tests were carried out at four different stages of the mosquito life cycle: L4 larvae (7 days posthatching), newly emerged adults, young adults and old adults (1, 7 and 14 days post-emergence, respectively). In Experiment 1, at each time step, either 20 larvae or 30 adults (15 males and 15 females) per tray (or, in the case of adults, cage) were haphazardly sampled from the pool of individuals. In Experiment 2 we sampled either 40 larvae or 80 adults (40 males and 40 females). The higher replication number was to account for the fact that this experiment had to be done blind: assignation to a particular insecticide resistance or susceptible genotype was done a posteriori (see "Mosquito genotyping" below). Since females emerged on average one day later than males, we respected a one-day shift in the sampling of males and females. Mosquito size was recorded by measuring (i) the thorax width of larvae and (ii) wing size of adults, using a binocular microscope linked to an image analysis software.

\section{Measures of phenoloxidase activity}

Melanization results from the enzymatic activation of prophenoloxidases (proPO) into phenoloxidases (PO), which initiate the production of melanin. Both the ready-to use PO (henceforth "active PO") and its proPO precursor are stored in the haemolymph. While the quantification of the active PO can be assessed directly (see below), the estimation of the overall investment in the PO pathway (henceforth "total PO") requires the prior transformation of proPO into PO using chymotrypsin. Biologically speaking, "active PO" measures the amount of PO naturally activated, while "total PO" measures the overall investment in this immune pathway (i.e. active PO plus inactive proPO, see also [37]).

As the amount of haemolymph that can be extracted from a single Culex mosquito is very limited, phenoloxidase activity was quantified using individual homogenates. As there is increasing evidence that in mosquitoes haemocytes in the haemolymph are the main source of proPO/ PO synthesis [38,39], this is unlikely to have biased our results. For this purpose, mosquitoes (larvae and adults) were crushed individually in $35 \mu \mathrm{L}$ of cold PBS buffer. After centrifugation $\left(2 \mathrm{~min}, 4^{\circ} \mathrm{C}, 3000 \mathrm{rpm}\right), 15 \mu \mathrm{L}$ of the supernatant was collected, immediately frozen in liquid nitrogen and stored at $-80^{\circ} \mathrm{C}$ for subsequent analysis.

The analyses of the active PO and total PO activities were carried out using a spectrophotometric assay [37]. The assay was performed using $5 \mu \mathrm{L}$ of supernatant extract added to a microplate well containing $20 \mu \mathrm{L}$ of PBS buffer and either $140 \mu \mathrm{L}$ of $\mathrm{dH}_{2} \mathrm{O}$ to measure active PO, or $140 \mu \mathrm{L}$ of chymotrypsin solution (Sigma C-7762, $0.07 \mathrm{mg} / \mathrm{mL}$ of $\mathrm{dH}_{2} \mathrm{O}$ ) to measure total $\mathrm{PO}$ activity. In both cases, $20 \mu \mathrm{L}$ of L-Dopa solution (the substrate for the PO, Sigma D-9628, $4 \mathrm{mg} / \mathrm{mL}$ of $\mathrm{dH}_{2} \mathrm{O}$ ) was added to the mixture and the colorimetric reaction was followed in a microplate reader (Versamax, Molecular Devices) at $30^{\circ} \mathrm{C}$. Readings were taken for $30 \mathrm{~min}$ at $490 \mathrm{~nm}$. Enzyme activity was analysed using the software SOFTMax Pro 5.2 (Molecular Devices) and measured as the slope $\left(\mathrm{V}_{\max }\right.$ value) of the reaction curve during the linear phase. The values reported refer to the activity present in a standard $1 \mu \mathrm{L}$ volume of supernatant. In experiment 1 , total and active PO were highly correlated and provided nearly identical results (see below). Due to a technical problem (faulty chymotrypsin), however, we were unable to reliably quantify total PO activity in experiment 2 .

\section{Mosquito genotyping}

Field-caught mosquitoes (experiment 2) need to be typed to determine their insecticide resistance status. 
Once the haemolymph was collected and analysed for PO activity (see above), the mosquito was homogenised and the genomic DNA was extracted using the procedure outlined in the DNeasy Blood \& Tissue Kit (Qiagen). Insecticide resistance through the overproduction of carboxylesterases was analysed using RFLP analysis at the Ester-3 gene [40]. This technique allows us to distinguish between susceptible mosquitoes (allele $\operatorname{Ester}^{\mathrm{O}}$ ), and the most common insecticide resistant carboxylesterase allozyme present in the study area: A4-B4 (allele Ester ${ }^{4}$ ). Insecticide resistance through the modification of the acetylcholinesterase was established using RFLP analysis at the ace-1 gene [41]. This technique allows us to distinguish between susceptible (allele ace- $1^{s}$ ) and targetsite resistant (allele ace- $1^{s}$ ) mosquitoes.

Overall, 824 individuals were successfully allocated to one of three groups depending on their insecticide resistant status (Additional file 1: Table S1): S (fully susceptible, Ester ${ }^{O}$, ace- $1^{s}$ ), E (resistant through esterase overproduction, Ester ${ }^{4}$, ace- $1^{s}$ ), and R (resistant through acetylcholinesterase modification, Ester ${ }^{0}$, ace- $\left.1^{R}\right)$. We failed to obtain a reliable genotype for 165 samples, and 130 other samples were discarded because of mixed resistance or rare Ester-3 genotypes (A1, A2 or a mixedallele combination).

\section{Statistical analyses}

The statistical analyses were run using the $\mathrm{R}$ software (v. 2.14.0). Variation in phenoloxidase activity was analyzed using linear mixed-effect models (lme function, nlme package). Tray (larvae) or cage (adults) effects were included as random factors, and insecticide resistance (IR), stage (larval/adult) age (adults only), sex (adults only) and size as fixed factors. Models were simplified by sequentially eliminating the least non-significant term to obtain minimal adequate models using a standard procedure of likelihood comparison (using the function anova. lme and specifying a marginal type test). When appropriate, a posteriori contrasts were carried out by aggregating factor levels that did not significantly differ from each other and by testing the fit of the simplified model [42].

\section{Results}

\section{Experiment 1- Isogenic strain mosquitoes}

The total and active PO activities per $\mu \mathrm{L}$ homogenate were highly correlated $\left(r=0.97, t_{1}, \quad 1313=155.58, P<\right.$ $0.0001)$. Insecticide resistance affects both the active and total PO activities (active PO: $F_{2,232}=4.89, P=0.0083$; total PO: $\left.F_{2}, 232=6.70, P=0.0006\right)$ of mosquito larvae. Esterase resistant (SA4B4) larvae have significantly less active and total PO activities per $\mu \mathrm{L}$ than either susceptible (SLAB) or acetylcholinesterase resistant (SR) larvae (Figure 1a, b; contrast analysis SA4 vs SLAB + SR, active PO: $\chi_{1}^{2}=8.68, \quad P=0.0032$; total $P O: \chi_{1}^{2}=13.11, \quad P=$
0.0003). Larval size is positively related to both active PO $\left(F_{1,232}=94.73, P<0.0001\right)$ and total PO $\left(F_{1,232}=\right.$ 111.80, $P<0.0001)$, but this effect is independent of the insecticide resistance strain (IR*size interaction, active PO: $F_{2,230}=0.84, P=0.4342$; total PO: $F_{2,230}=0.57, P=$ 0.5665 ; the size of L4 larvae does not differ between strains, $\left.F_{2,233}=0.35, P=0.7055\right)$.

Adult emergence is characterized by an intense reduction of the phenoloxidase activity (Figure 1a, b). Larvae (mean \pm se, active PO: $6.34 \pm 0.12$, total PO: $6.71 \pm 0.12$ ) have roughly more than twice the amount of active PO $\left(2.20 \pm 0.06\right.$; stage effect $\left.F_{1,590}=1037.46, P<0.0001\right)$ and total PO $\left(2.85 \pm 0.07 ; F_{1,590}=776.16, P<0.0001\right)$ per $\mu \mathrm{l}$ homogenate than newly emerged adults (analysis carried out pooling together newly emerged males and females, see Figure 1a, b).

The full model containing all explanatory factors rendered a very significant three way $I R$ *age sex interaction for adult active and total PO activities $\left(F_{4,1055}=4.87\right.$, $P=0.0007$ and $F_{4,1055}=4.96, P=0.0006$, respectively; Additional file 1: Table S2). In order to investigate this three-way interaction in more detail we decomposed it into two separate analyses (Tables 1 and 2).

In the first analysis, we pooled all IR strains together and we investigated the effect of mosquito age and sex on PO activity. This analysis shows that females have significantly higher active and total PO activities per $\mu \mathrm{L}$ homogenate than males (Table 1a). The difference between the sexes is particularly striking on days 1 and 7, but tends to decrease later in life (day 14, Figure 1a, b). In both males and females, PO decreases as the mosquitoes age, but the pattern of reduction is markedly different between the sexes (significant age sex interaction, Table 1a). Males show a monotonic decrease in PO activity as they age. In contrast, female active and total PO activities are maintained until day 7 before decreasing drastically on day 14 .

In the second analysis we explored the effect of insecticide resistance and age on PO activity separately for males and females (Table 2a). In both sexes, the pattern of PO decay is markedly different between the insecticideresistant and susceptible strains (significant $I R^{*}$ age interaction, Table 2a). In 1-day old adult females, there was a trend towards both of the insecticide resistant strains (SA4 and SR) having higher PO levels than their susceptible (SLAB) counterparts, although this trend was only significant for the active PO (active PO: $\chi_{1}^{2}=5.74, P=$ 0.0166; total PO: $\chi_{1}^{2}=3.95, P=0.1386$ ). In 7 and 14-day old females, however, it is only the esterase resistant (SA4B4) strain which has significantly higher levels of both total and active PO activity than SLAB and SR females (contrast analyses; active PO: day 7: $\chi_{1}^{2}=13.98$, $P=0.0002$; day 14: $\chi_{1}^{2}=248.84, P<0.0001$; total PO: day 7: $\chi_{1}^{2}=10.90, P=0.0010$; day $14: \chi_{1}^{2}=215.82, P<0.0001$; 
(a)

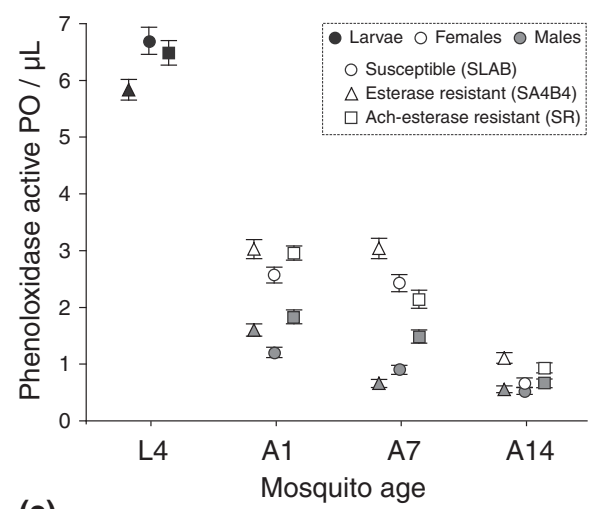

(c)

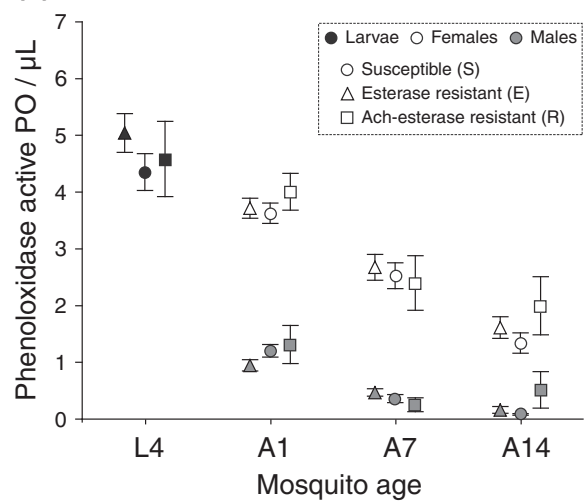

(b)

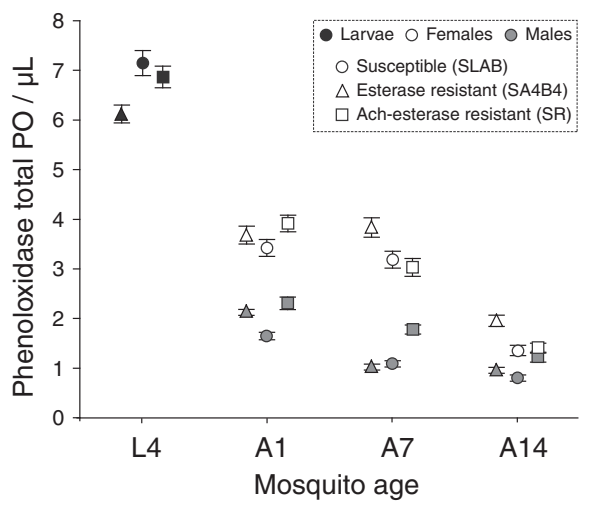

Figure 1 Levels of phenoloxidase activity (active PO and total PO) per microlitre of homogenate sample (mean \pm se) in unsexed L4 larvae (dark symbols), and in male (grey symbols) and female (white symbols) adults of Culex pipiens mosquitoes. Adults were either 1-day old (A1), 7-days old (A7) or 14-days old (A14). Experiment 1: (a) active PO and (b) total PO in isogenic strain mosquitoes: insecticide-susceptible mosquitoes (SLAB strain, circles), esterase resistant mosquitoes (SA4B4 strain, triangles), acetylcholinesterase resistant mosquitoes (SR strain, squares). Experiment 2: (c) active PO in field-caught mosquitoes: insecticide-susceptible mosquitoes ( $\mathrm{S}$, circles), esterase resistant mosquitoes ( $\mathrm{E}$, triangles), acetylcholinesterase resistant mosquitoes ( $R$, squares).

Figure 1a, b). In addition, whereas both SLAB and SA4B4 females maintained their level of PO activity for the first 7 days, SR females lost about $30 \%$ of their PO activity in the same period (Figure 1a, b). In 1-day old males the pattern was similar to that of females: resistant (SA4B4 and SR) strains had significantly higher PO activity levels than their susceptible (SLAB) counterparts (active
PO: $X_{1}^{2}=12.64, P<0.0001$; total $P O: X_{1}^{2}=14.17, P<$ $0.0001)$. However, in 7 and 14-day old males it is the acetylcholinesterase resistant (SR) strain which shows significantly higher PO levels than the other two strains (contrast analyses; active PO: day 7: $\chi_{1}^{2}=35.83, P=$ 0.0002; day 14 : $\chi_{1}^{2}=131.41, P<0.0001$; total PO: day 7 : $\chi_{1}^{2}=35.01, P<0.0001 ;$ day $14: X_{1}^{2}=135.25, P<0.0001$;

Table 1 Variation in phenoloxidase activity of adult Culex pipiens mosquitoes according to age, sex and size

\begin{tabular}{|c|c|c|c|c|c|c|}
\hline \multirow[b]{3}{*}{ Factor } & \multicolumn{4}{|c|}{ (a) Isogenic mosquitoes } & \multirow{2}{*}{\multicolumn{2}{|c|}{$\begin{array}{c}\text { (b) Wild mosquitoes } \\
\text { Active PO }\end{array}$}} \\
\hline & \multicolumn{2}{|c|}{ Active PO } & \multicolumn{2}{|c|}{ Total PO } & & \\
\hline & $F_{(\mathrm{df})}$ & $P$ & $F_{\text {(df) }}$ & $P$ & $F_{(\mathrm{df})}$ & $P$ \\
\hline Age & $247.17(2,1065)$ & $<0.0001$ & $214.62(2,1065)$ & $<0.0001$ & $111.15(2,734)$ & $<0.0001$ \\
\hline Sex & $29.43(1,1065)$ & $<0.0001$ & $15.10(1,1065)$ & 0.0001 & $1.24_{(1,734)}$ & 0.2661 \\
\hline Size & $4.87(1,1065)$ & 0.0275 & $0.31(1,1065)$ & 0.5745 & $20.71(1,734)$ & $<0.0001$ \\
\hline Age $^{*} \operatorname{sex}$ & $58.25(2,1065)$ & $<0.0001$ & $51.78(2,1065)$ & $<0.0001$ & $19.66(2,734)$ & $<0.0001$ \\
\hline Sex*size & $22.33(1,1065)$ & $<0.0001$ & $10.39(1,1065)$ & 0.0013 & $3.84_{(1,734)}$ & 0.0504 \\
\hline
\end{tabular}

(a) Experiment 1 using isogenic strain mosquitoes. (b) Experiment 2 using wild mosquitoes. 
Table 2 Variation in phenoloxidase activity of adult female and male Culex pipiens mosquitoes according to insecticide resistance genotype (IR), age and size

\begin{tabular}{|c|c|c|c|c|c|c|}
\hline \multirow[b]{3}{*}{ Factor } & \multicolumn{4}{|c|}{ (a) Isogenic mosquitoes } & \multirow{2}{*}{\multicolumn{2}{|c|}{$\begin{array}{c}\text { (b) Wild mosquitoes } \\
\text { Active PO }\end{array}$}} \\
\hline & \multicolumn{2}{|c|}{ Active PO } & \multicolumn{2}{|c|}{ Total PO } & & \\
\hline & $F_{\text {(df) }}$ & $P$ & $F_{\text {(df) }}$ & $P$ & $F_{\text {(df) }}$ & $P$ \\
\hline \multicolumn{7}{|l|}{ Females } \\
\hline $\mathbb{R}$ & $3.22(2,525)$ & 0.0409 & $2.62(2,525)$ & 0.0735 & $0.27_{(2,348)}$ & 0.7658 \\
\hline Age & $67.99(2,525)$ & $<0.0001$ & $47.62(2,525)$ & $<0.0001$ & $25.79(2,348)$ & $<0.0001$ \\
\hline Size & $0.02(1,525)$ & 0.8845 & $4.19_{(1,525)}$ & 0.0412 & $12.13(1,348)$ & 0.0006 \\
\hline $\mathbb{R}^{*}$ age & $2.66(4,525)$ & 0.0319 & $3.39(4,525)$ & 0.0094 & $0.45(4,348)$ & 0.7756 \\
\hline \multicolumn{7}{|l|}{ Males } \\
\hline $\mathbb{R}$ & $10.71_{(2,525)}$ & $<0.0001$ & $10.29(2,525)$ & $<0.0001$ & $3.22(2,371)$ & 0.0409 \\
\hline Age & $40.31(2,525)$ & $<0.0001$ & $42.32(2,525)$ & $<0.0001$ & $24.71(2,371)$ & $<0.0001$ \\
\hline Size & $12.64(1,525)$ & 0.0004 & $19.85(1,525)$ & $<0.0001$ & $5.25(1,371)$ & 0.0225 \\
\hline $\mathrm{IR}^{*}$ age & $4.07(4,525)$ & 0.0029 & $2.90(4,525)$ & 0.0215 & $2.41_{(4,371)}$ & 0.0490 \\
\hline
\end{tabular}

(a) Experiment 1 using isogenic strain mosquitoes. (b) Experiment 2 using wild mosquitoes.

Figure 1a, b). Whereas PO activity in SLAB and SR males decreased linearly with time, it was halved in SA4 males between day 1 and day 7 .

\section{Experiment 2- Field-caught mosquitoes}

Due to a technical problem (see above), in experiment 2, only active PO measurements could be obtained. The active PO of field-caught larvae is identical for the three insecticide resistance genotypes $\left(F_{2}, 72=1.05, P=0.3565\right.$; Figure 1c). Larval size is positively correlated with active PO $\left(F_{1}, 72=65.08, P<0.0001\right)$, but this relationship is not significantly affected by the insecticide resistance genotype ( $I R *$ size interaction, $F_{1,70}=2.67, P=0.0763$; the size of L4 larvae does not differ between strains, $F_{2,73}=$ $1.34, P=0.2690)$.

As was the case for the isogenic strain mosquitoes, the transition from larvae to newly emerged adult is characterized by a large (2-fold) reduction in the amount of active PO per $\mu \mathrm{L}$ homogenate (larvae $4.77 \pm 0.22$, adults A1 $2.41 \pm 0.11$; stage effect $F_{1,315}=108.02, P<0.0001$; analysis was carried out pooling together newly emerged males and females, see Figure 1c).

Also congruent with the isogenic strain experiments is the fact that females have significantly higher levels of active PO per $\mu \mathrm{L}$ homogenate than males (Table $1 \mathrm{~b}$ ). In both males and females, PO decreases significantly as the mosquitoes age, although the pattern of reduction differs between the sexes (significant age ${ }^{*}$ sex interaction, Table $1 \mathrm{~b}$ ). The decreased PO activity with age was stronger for females than for males (Figure 1c).

We explored the effect of insecticide resistance and adult age on PO immune activity separately for males and females (Table 2b). In both sexes, active PO decreases significantly with age (Table $2 \mathrm{~b}$ ). The most striking difference with the isogenic strain experiments is that here insecticide resistance does not have any noticeable effect on PO immune activity (Figure 1c).

\section{Discussion}

\section{Insecticide resistance}

In insects, the evolution of insecticide resistance has often been found to be associated to life history costs (see however [43]). These costs are largely expected to appear in mosquitoes that are resistant to insecticides through the overproduction of detoxifying enzymes, because of the large amount of resources that need to be diverted for the large-scale production of the extra proteins [2]. Consistent with this, we and others have shown that $C x$. pipiens females resistant to insecticides through the overproduction of esterases have $30 \%$ less energetic resources [13], a higher pre-imaginal mortality [44] and a significantly shorter lifespan $[45,46]$ than susceptible ones. A reasonable prediction is therefore that these mosquitoes may also be less immunocompetent than their susceptible counterparts, which if true, could have dramatic consequences for the transmission of diseases. The PO activity of our field-caught esterase-resistant and acetylcholinesterase-resistant females was, however, equal to that of susceptible females. More surprisingly, the results of the isogenic laboratory strains showed that insecticide resistant mosquitoes tend to have higher, as opposed to lower PO activities. In particular, young (7-day) and, to a lesser extent, old (14-day) esteraseresistant (SA4B4) adult females had a significantly higher PO activity than individuals of the other two strains. The same is true for young and old acetylcholinestraseresistant males, although the reasons for the differences between the sexes are not entirely clear. Intriguingly, these unexpected results are in agreement with several other studies comparing the immunity of insecticide 
resistant and susceptible mosquito populations using laboratory-selected strains. One straightforward explanation is that the strong insecticide doses used to select for resistance in the laboratory selects for insects with higher PO levels because PO plays a key role in cuticle sclerotization, and as the cuticle is a major route for insecticide penetration, insecticide resistance is often associated to thicker/harder cuticles $[47,48]$. Contrary to this hypothesis is the fact that no such increased PO activity was observed in acetylcholinesterase-resistant females nor in esterase-resistant males, despite them being subject to similarly high insecticide pressures. Furthermore, the increased immune activity of insecticide resistant insects is not restricted to PO. Using the same $C x$. pipiens laboratory strains, we recently quantified the antibacterial peptide and nitric oxide synthase (NOS) transcripts of 7-day old insecticide resistant and susceptible Cx. pipiens females either before (constitutive) or after (induced) an LPS challenge. Insecticide resistant isogenic strains were shown to have a significantly enhanced expression of virtually all the immune related genes investigated [10]. These results agreed with previous microarray studies carried out in two other species: Anopheles gambiae [49] and An. stephensi [47], where it was also shown that several antibacterial peptides and the NOS gene were constitutively transcribed at a higher level in laboratory-maintained insecticide-resistant strains of mosquitoes than in their insecticide susceptible counterparts. One pervasive criticism addressed at mRNA quantification studies is, however, that they do not necessarily reflect the level of immunity of the organism, as regulation can take place downstream from the transcription step [50]. The results of the present study show that the pleiotropic effects of insecticide resistance on the immunity of laboratory-selected mosquitoes are also seen at the level of an immune effector (PO).

The mechanisms underlying the higher PO activity in esterase-overproducing laboratory females are not clear, but at least three potential scenarios are possible. Esterase overproduction in $C x$. pipiens is affected through the co-amplification of two paralogous esterase loci: Est-3 (encoding for the esterase A) and Est-2 (esterase B). The amplicons on which these esterases occur are, however, much larger $(30-60 \mathrm{~kb})$ than the esterase containing region $(\sim 10 \mathrm{~kb},[11])$. So far, only one other gene present in the amplicon of insecticide resistant $C x$. pipiens has been identified (aldehyde oxydase, [11]) but there could be others. One possibility is that one of the immune genes implicated in the complex enzymatic cascade that leads to the activation of PO [23] or one of their trans-acting regulatory genes, is coamplified with the esterases. Alternatively, the original esterase resistance allele used to create the SA4B4 strain [33,51] may have been in linkage disequilibrium with genes involved in the melanisation cascade. Such associations have been found before. For example, in a particular strain of An. gambiae, the genes responsible for Plasmodium melanization seem to be in the same chromosomal inversion as an esterase locus that encodes for a protein very similar to the insecticideconferring carboxylase from $C x$. pipiens [52].

Finally, it is not impossible that the strong immune phenotype observed in the isogenic SA4B4 strain may be the result of epistatic interactions between the insecticide resistance genes (or genes closely associated with them at the amplicon or allelic level), and the SLAB genetic background. The finding, however, that selection for high resistance levels in laboratory strains from two other mosquito species also results in an upregulation of the immune system $[47,49]$ suggests that our results are not specific to a particular genetic background and that the effect may be a common artefact of laboratory strains.

Irrespective of the underlying mechanism, our results add weight to claims that the results of experiments using laboratory-selected insecticide-resistant strains, still the most widely used method to investigate the pleiotropic effects of insecticide resistance, must be interpreted with caution $[2,34,53]$ and, whenever possible, experiments should be done using wild-caught mosquitoes. Unfortunately, due to the long and complex history of insecticide use in most mosquito-infected areas, which result in most individuals being resistant at, at least, one of the many possible insecticide resistant loci, the opportunities for comparing field-caught sympatric insecticide resistant and susceptible mosquitoes are few and far between, so the options for carrying meaningful comparative studies are limited. On the other hand, the greater genetic and environmental variances and the constraints on sample sizes that are inevitably associated with wild-caught mosquitoes (apparent in our experiment by smaller sample sizes and larger error bars in our field-caught acetylcholinesteraseresistant mosquitoes) may reduce the statistical power to detect a difference.

\section{Mosquito stage, sex and age}

Laboratory and field-caught mosquitoes were consistent in showing that: (i) larvae have a higher PO activity than adults, (ii) adult females have a higher PO activity than males and (iii) PO activity declines with adult age.

The process of metamorphosis that takes place in holometabolous insects has often been considered to be an adaptive strategy to decouple the life history traits of larvae and adults [54]. Recent work, however, shows that, at least for the immune system, there is a certain degree of continuity between the larval and adult stages. For example, Fellous and Lazzaro [55] have shown a great degree of genetic correlation in the transcription of certain antibacterial peptides between the larval and adult stages of Drosophila melanogaster. Other studies have shown 
that the larval conditions determine the immunity and vectorial capacity of the adults [56-58]. Despite this, we know surprisingly little about larval immunity in mosquitoes (but see [59]). One general prediction is that larvae should have a stronger immune system than adults because their aquatic life style exposes them to a higher density and diversity of parasites. Cx. pipiens larvae can be found in a fairly wide range of larval habitats but are generally associated with water that has a high organic and bacterial content (such as sewage treatment plants). The high PO activity we found in both the laboratory and the field-caught larvae may be consistent with this hypothesis. Interestingly, in the isogenic laboratory strains, the PO activity per $\mu \mathrm{L}$ homogenate was twice as high in larvae as in adults (Figure 1a, b). The effect was, however, less drastic in field-caught mosquitoes, particularly in females (Figure 1c), a result that may be explained by the fact that wild females are exposed to a larger density and diversity of blood-associated parasites than laboratory-reared ones. Given that PO activities were assessed from crushed extracts and that we could not have a common size estimator for larvae and adults (i.e. thorax width and wing size, respectively), the comparison of immune activity between mosquito stages needs to be taken with caution.

The difference in immune investment we found between males and females agrees with previous data from other invertebrates $[60,61]$. This can be explained by two non-exclusive factors. First, by differences in lifespan and in the constraints associated with their respective reproductive schedules [62,63]. Indeed, males of most species, including mosquitoes, have what Zuk [64] described as a "live hard, die young" strategy. In contrast, the female reproductive life is made up of lengthy gonotrophic cycles, each of them consisting of a period of host seeking, followed by blood feeding, egg maturation and oviposition which, collectively, can last 2-5 days depending on the species [65]. For females, therefore, longevity is an essential component of their reproductive fitness and surviving parasites is, by extension, potentially much more important. Secondly, by sex-associated differences in parasite exposure. While $C x$. pipiens males feed exclusively on nectar [65], which plants keep mostly bacterial and fungifree [66], females are exposed to a wide array of bloodassociated pathogens that include viruses (West Nile and encephalitis), protozoan (Plasmodium, Trypanosoma) and metazoan (Wuchereria) parasites $[3,67,68]$. To verify that these a posteriori explanations for our results hold, an interesting experiment would, therefore, be to compare the immune competence in males and females of species, such as the tsetse fly (Glossina sp.), where both sexes blood feed and have similar lifespans [69]. Despite using whole body extracts to quantify the PO activity, the difference between males and females found here are unlikely to have been biased by the presence of ovaries in the mixture. PO does indeed play a key role in egg production, but this role seems to be restricted to the tanning of the chorion, which is secreted just before the eggs are laid [39]. Previous reports of PO activity in other tissues such as ovaries may, in fact, be due to contamination with haemocytes [38,39]. In mosquitoes, $\mathrm{PO}$ in the ovaries is therefore largely associated to the presence of mature (chorionated) eggs. However, since in our experiment mosquitoes were not allowed to blood feed, they did not contain any mature eggs in the ovarioles.

Although the connection between age and immunocompetence has been well established in insects, its occurrence in mosquitoes has been largely ignored or given only cursory attention [70,71], and this despite earlier reports showing that parasite infection intensity is higher older mosquitoes [72,73]. Our results show that, in female mosquitoes in particular, PO activity declines markedly with age, so that by day 14 females have lost between two thirds (isogenic mosquitoes) and a half (field mosquitoes) of their PO at emergence (Figure 1a, b and c). Such immune senescence is less marked in males possibly because of the lower PO levels at emergence that does not leave much room for further reductions. Aging has been previously associated with a decrease in PO activity and/or a decreased ability to encapsulate parasites in several insect species [71,73-79]. The decrease in PO with age may not, however, necessarily indicate a generalized immune senescence but be a result of a plastic adjustment of immune function with age. Moret and Schmid-Hempel [80], for example, found that in bumble bees PO and antimicrobial activity strongly trade-off with each other: as colonies age the decrease in PO activity is associated with an increase in antimicrobial activity, even in the presence of plentiful resources. In mosquitoes, however, no such age-associated change has been detected for antimicrobial peptide production [71]. In addition, the evidence available suggests that in mosquitoes the melanisation and antibacterial responses are positively correlated [28].

Immune decay has been described in many invertebrate species, but its consequences are particularly poignant for mosquitoes because only relatively old females transmit diseases. This correlation between mosquito age and parasite transmission is due to the combined effects of (1) a long time lag between adult emergence and the activation of the host seeking behaviour (between 2-5 days for most species, [65]), (2) the interval between blood feeding and laying events imposed by the gonotrophic cycles (2-4 days, [65]), and (3) the long extrinsic incubation period of most mosquito-transmitted parasites (10-14 days for filaria, West Nile and encephalitis viruses, [81-83]). 


\section{Conclusions}

Mosquito immunocompetence cannot be reduced to a single immunological measurement $[84,85]$. However, the PO cascade is a key component of insect host condition, hemostasis and immunity and is undoubtedly a major player in the fight against a wide range of parasites [17]. We found no effect of insecticide resistance on the PO activity of field-caught $C x$. pipiens mosquitoes. Further work is needed to determine whether these results extend to other mosquito species, immune effectors and insecticide resistant mechanisms, and to examine the consequences of insecticide resistance on vector competence to pathogens $[36,86]$. We also observed a significant decline in $\mathrm{PO}$ activity with mosquito age. Despite its potential consequences for parasite transmission, immune senescence in mosquitoes has been little studied (but see $[71,73,76]$ ). Assuming that PO measurements correlate with disease resistance, our results predict that, everything else being equal, older mosquitoes may show a higher prevalence and/or intensity of infection than younger, more immunocompetent ones. We are, however, not aware of any studies that have compared the vectorial capacity of mosquitoes of different ages and further work is needed to demonstrate an association between levels of PO activity and resistance to parasitism in mosquitoes (especially wild-caught mosquitoes). In addition, an interesting spin-off of these results is that late life acting (or "evolution proof") insecticides, whose purpose is to kill mosquitoes after they have reproduced but before they are able to transmit parasites $[87,88]$ would have the additional advantage of killing the least immune competent (and thus, potentially, most transmission-efficient) fraction of the mosquito population.

\section{Additional file}

\section{Additional file 1: Supplementary materials. Table S1: Mosquito} sample size of the different insecticide resistance groups (insecticidesusceptible mosquitoes (SLAB/S), esterase resistant mosquitoes (SA4B4/E), acetylcholinesterase resistant mosquitoes (SR/R) according to stage (larvae, adults), age $(1,7,14$ days) and sex (males, females). (a) Experiment 1 using isogenic strain mosquitoes. (b) Experiment 2 using wild mosquitoes. Table S2: Variation in phenoloxidase activity of adult Culex pipiens mosquitoes according to insecticide resistance (IR), age and sex. (a) Experiment 1 using isogenic strain mosquitoes. (b) Experiment 2 using wild mosquitoes.

\section{Competing interests}

The authors declare that they have no competing interests.

\section{Authors' contributions}

SC, SG and AR conceived and designed the experiment. SC performed the experiment and analysed the data. SC, SG and AR wrote the paper. All authors read and approved the final version of the manuscript.

\section{Acknowledgments}

The authors would like to thank M. Weill and P. Makoundou (ISEM) for providing us with the mosquito isogenic lines, A. Nicot for helping in the laboratory and C. Chevillon and N. Pasteur for useful discussions. AR acknowledges financial support from the CNRS, and an ANR SEST "IRMAL" grant. SG acknowledges financial support from the CNRS, an ANR JCJC "EPICE" grant, and an European Research Council Starting Grant 243054 "EVOLEPID".

\section{Author details}

${ }^{1}$ Maladies Infectieuses et Vecteurs: Ecologie, Génétique, Evolution et Contrôle (MIVEGEC), UMR CNRS 5290-IRD 224-UM1-UM2, Montpellier, France. ${ }^{2}$ Centre d'Ecologie Fonctionnelle et Evolutive (CEFE), UMR CNRS 5175, Montpellier, France. ${ }^{3}$ MIVEGEC, Institut de Recherche pour le Développement, 911 av. Agropolis, 34394, Montpellier, France.

Received: 8 July 2013 Accepted: 23 October 2013

Published: 31 October 2013

\section{References}

1. Hemingway J, Ranson $\mathrm{H}$ : Insecticide resistance in insect vectors of human disease. Annu Rev Entomol 2000, 45:371-391.

2. Rivero A, Vezilier J, Weill M, Read AF, Gandon S: Insecticide control of vector-borne diseases: when is insecticide resistance a problem? PLOS Pathog 2010, 6:e1001000.

3. McCarroll L, Paton MG, Karunaratne S, Jayasuryia HTR, Kalpage KSP Hemingway J: Insecticides and mosquito-borne disease. Nature 2000 407:961-962.

4. Yassine H, Osta MA: Anopheles gambiae innate immunity. Cell Microbiol 2010, 12:1-9.

5. Aliota MT, Fuchs JF, Mayhew GF, Chen CC, Christensen BM: Mosquito transcriptome changes and filarial worm resistance in Armigeres subalbatus. BMC Genomics 2007, 8:463.

6. Sanchez-Vargas I, Scott JC, Poole-Smith BK, Franz AWE, Barbosa-Solomieu V, Wilusz J, Olson KE, Blair CD: Dengue virus type 2 infections of Aedes aegypti are modulated by the mosquito's RNA interference pathway. PLOS Pathog 2009, 5:e1000299.

7. Dimopoulos G: Insect immunity and its implication in mosquito-malaria interactions. Cell Microbiol 2003, 5:3-14.

8. Michel K, Kafatos FC: Mosquito immunity against Plasmodium. Insect Biochem Mol Biol 2005, 35:677-689.

9. Hemingway J, Hawkes NJ, McCarroll L, Ranson H: The molecular basis of insecticide resistance in mosquitoes. Insect Biochem Mol Biol 2004, 34:653-665.

10. Vézilier J, Nicot A, De Lorgeril J, Gandon S, Rivero A: The impact of insecticide resistance on Culex pipiens immunity. Evol Appl 2013. in press.

11. Hemingway J, Coleman M, Paton M, McCarroll L, Vaughan A, DeSilva D: Aldehyde oxidase is coamplified with the World's most common Culex mosquito insecticide resistance-associated esterases. Insect Mol Biol 2000, 9:93-99.

12. Romans P, Black WC, Sakai RK, Gwadz RW: Linkage of a gene causing malaria refractoriness to Diphenol oxidase-A2 on chromosome 3 of Anopheles gambiae. Am J Trop Med Hyg 1999, 60:22-29.

13. Rivero A, Magaud M, Nicot A, Vézillier J: Energetic cost of insecticide resistance in Culex pipiens mosquitoes. J Med Entomol 2011, 48:694-700.

14. Schmid-Hempel P: Variation in immune defence as a question of evolutionary ecology. Proc R Soc Lond B Biol Sci 2003, 270:357-366.

15. Siva-Jothy MT, Moret $Y$, Rolff J: Insect immunity: an evolutionary ecology perspective. Adv Insect Physiol 2005, 32:1-48.

16. Raymond M, Berticat C, Weill M, Pasteur N, Chevillon C: Insecticide resistance in the mosquito Culex pipiens: what have we learned about adaptation ? Genetica 2001, 112:287-296.

17. Christensen BM, Li JY, Chen CC, Nappi AJ: Melanization immune responses in mosquito vectors. Trends Parasitol 2005, 21:192-199.

18. Zhao PC, Lu ZQ, Strand MR, Jiang HB: Antiviral, anti-parasitic, and cytotoxic effects of 5,6-dihydroxyindole (DHI), a reactive compound generated by phenoloxidase during insect immune response. Insect Biochem Mol Biol 2011, 41:645-652.

19. King JG, Hillyer JF: Infection-induced interaction between the mosquito circulatory and immune systems. PLoS Pathog 2012, 8:e1003058.

20. Rodriguez-Andres J, Rani S, Varjak M, Chase-Topping ME, Beck MH, Ferguson MC, Schnettler E, Fragkoudis R, Barry G, Merits A, et al: Phenoloxidase 
activity acts as a mosquito innate immune response against infection with Semliki Forest Virus. PLoS Pathog 2012, 8:e1002977.

21. Yassine $H$, Kamareddine L, Osta MA: The mosquito melanization response is implicated in defense against the entomopathogenic fungus Beauveria bassiana. PLoS Pathog 2012, 8:e1003029.

22. Santa-Ana M, Capela RA, Christensen BM: Reproductive costs of the immune response of the autogenous mosquito Culex pipiens molestus against inoculated Dirofilaria immitis. Vector-Borne Zoo Dis 2007, 7:541-546.

23. Cerenius L, Söderhäll K: The prophenoloxidase-activating system in invertebrates. Immunol Rev 2004, 198:116-126.

24. Nappi AJ, Christensen BM: Melanogenesis and associated cytotoxic reactions: applications to insect innate immunity. Insect Biochem Mol Biol 2005, 35:443-459.

25. Christophides GK, Zdobnov E, Barillas-Mury C, Birney E, Blandin S, Blass C, Brey PT, Collins FH, Danielli A, Dimopoulos G, et al: Immunity-related genes and gene families in Anopheles gambiae. Science 2002, 298:159-165.

26. Waterhouse RM, Kriventseva EV, Meister S, Xi ZY, Alvarez KS, Bartholomay LC, Barillas-Mury C, Bian GW, Blandin S, Christensen BM, et al: Evolutionary dynamics of immune-related genes and pathways in disease-vector mosquitoes. Science 2007, 316:1738-1743.

27. Cotter SC, Kruuk LEB, Wilson K: Costs of resistance: genetic correlations and potential trade-offs in an insect immune system. J Evol Biol 2004, 17:421-429.

28. Lambrechts L, Vulule J, Koella JC: Genetic correlation between melanization and antibacterial immune responses in a natural population of the malaria vector Anopheles gambiae. Evolution 2004 58:2377-2381

29. Meredith JM, Hurd H, Lehane MJ, Eggleston P: The malaria vector mosquito Anopheles gambiae expresses a suite of larval-specific defensin genes. Insect Mol Biol 2008, 17:103-112.

30. Warr E, Aguilar R, Dong YM, Mahairaki V, Dimopoulos G: Spatial and sexspecific dissection of the Anopheles gambiae midgut transcriptome. Bmc Genomics 2007, 8:e37.

31. Martin LB, Weil ZM, Nelson RJ: Refining approaches and diversifying directions in ecoimmunology. Integr Comp Biol 2006, 46:1030-1039.

32. Glunt KD, Thomas MB, Read AF: The effects of age, exposure history and malaria infection on the susceptibility of Anopheles mosquitoes to low concentrations of pyrethroid. Plos One 2011, 6:e24968.

33. Berticat C, Boquien G, Raymond M, Chevillon C: Insecticide resistance genes induce a mating competition cost in Culex pipiens mosquitoes. Genet Res 2002, 79:41-47.

34. Curtis CF: Insecticide resistance and mosquito-borne disease. Lancet 2001, 357:656-656.

35. Lenormand T, Bourguet D, Guillemaud T, Raymond M: Tracking the evolution of insecticide resistance in the mosquito Culex pipiens. Nature 1999, 400:861-864.

36. Vézilier J, Nicot A, Gandon S, Rivero A: Insecticide resistance and malaria transmission: infection rate and oocyst burden in Culex pipiens mosquitoes infected with Plasmodium relictum. Malaria J 2010, 9:379.

37. Cornet $S$, Biard C, Moret Y: Variation in immune defence among populations of Gammarus pulex (Crustacea: Amphipoda). Oecologia 2009, 159:257-269.

38. Cui L, Luckhart S, Rosenberg R: Molecular characterization of a prophenoloxidase cDNA from the malaria mosquito Anopheles stephensi. Insect Mol Biol 2000, 9:127-137.

39. Kim SR, Yao R, Han Q, Christensen BM, Li J: Identification and molecular characterization of a prophenoloxidase involved in Aedes aegypti chorion melanization. Insect Mol Biol 2005, 14:185-194.

40. Berticat C, Dubois MP, Marquine M, Chevillon C, Raymond M: A molecular test to identify resistance alleles at the amplified esterase locus in the mosquito Culex pipiens. Pest Manag Sci 2000, 56:727-731.

41. Weill M, Malcolm C, Chandre F, Mogensen K, Berthomieu A, Marquine M, Raymond M: The unique mutation in ace-1 giving high insecticide resistance is easily detectable in mosquito vectors. Insect Mol Biol 2004, 13:1-7.

42. Crawley MJ: The R Book. England: John Wiley \& Sons, Ltd; 2007.

43. McCart $\mathrm{C}$, Buckling $\mathrm{A}$, ffrench-Constant $\mathrm{RH}$ : DDT resistance in flies carries no cost. Curr Biol 2005, 15:R587-R589.

44. Duron O, Labbe P, Berticat C, Rousset F, Guillot S, Raymond M, Weill M: High Wolbachia density correlates with cost of infection for insecticide resistant Culex pipiens mosquitoes. Evolution 2006, 60:303-314.
45. Agnew P, Berticat C, Bedhomme S, Sidobre C, Michalakis Y: Parasitism increases and decreases the costs of insecticide resistance in mosquitoes. Evolution 2004, 58:579-586.

46. Vézilier J, Nicot A, Gandon S, Rivero A: Plasmodium infection decreases fecundity and increases survival of mosquitoes. Proc R Soc B Biol Sci 2012, 279:4033-4041.

47. Vontas J, David JP, Nikou D, Hemingway J, Christophides GK, Louis C, Ranson $\mathrm{H}$ : Transcriptional analysis of insecticide resistance in Anopheles stephensi using cross-species microarray hybridization. Insect Mol Biol 2007, 16:315-324.

48. Wood OR, Hanrahan S, Coetzee M, Koekemoer LL, Brooke BD: Cuticle thickening associated with pyrethroid resistance in the major malaria vector Anopheles funestus. Parasit Vectors 2010, 3:67.

49. Vontas J, Blass C, Koutsos AC, David JP, Kafatos FC, Louis C, Hemingway J, Christophides GK, Ranson H: Gene expression in insecticide resistant and susceptible Anopheles gambiae strains constitutively or after insecticide exposure. Insect Mol Biol 2005, 14:509-521.

50. Bartholomay LC, Fuchs JF, Cheng LL, Beck ET, Vizioli J, Lowenberger C, Christensen BM: Reassessing the role of defensin in the innate immune response of the mosquito, Aedes aegypti. Insect Mol Biol 2004, 13:125-132.

51. Poirié $M$, Raymond $M$, Pasteur $N$ : Identification of two distinct amplifications of the Esterase-B locus in Culex pipiens ( $L$ ) mosquitoes from Mediterranean countries. Biochem Genet 1992, 30:13-26.

52. Collins FH, Paskewitz SM, Crews-Oyen AE: A genetic study of Plasmodium susceptibility in the African malaria vector Anopheles gambiae. Ann Soc Belg Med Trop 1991, 71(Suppl 1):225-232.

53. McCarroll L, Hemingway J: Can insecticide resistance status affect parasite transmission in mosquitoes? Insect Biochem Mol Biol 2002, 32:1345-1351.

54. Moran NA: Adaptation and constraint in the complex life-cycles of animals. Annu Rev Ecol Syst 1994, 25:573-600.

55. Fellous S, Lazzaro BP: Potential for evolutionary coupling and decoupling of larval and adult immune gene expression. Mol Ecol 2011, 20:1558-1567.

56. Fellous S, Lazzaro BP: Larval food quality affects adult (but not larval) immune gene expression independent of effects on general condition. Mol Ecol 2010, 19:1462-1468.

57. Okech BA, Gouagna LC, Yan GY, Githure Jl, Beier JC: Larval habitats of Anopheles gambiae s.s. (Diptera: Culicidae) influences vector competence to Plasmodium falciparum parasites. Malaria J 2007, 6:50.

58. Bargielowski I, Koella JC: A possible mechanism for the suppression of Plasmodium berghei development in the mosquito Anopheles gambiae by the microsporidian Vavraia culicis. PLoS One 2009, 4:e4676.

59. Agianian B, Lesch C, Loseva O, Dushay MS: Preliminary characterization of hemolymph coagulation in Anopheles gambiae larvae. Dev Comp Immunol 2007, 31:879-888

60. Kurtz J, Sauer KP: Gender differences in phenoloxidase activity of Panorpa vulgaris hemocytes. J Invertebr Pathol 2001, 78:53-55.

61. Rolff J: Effects of age and gender on immune function of dragonflies (Odonata, Lestidae) from a wild population. Can J Zool 2001, 79:2176-2180.

62. Rolff J: Bateman's principle and immunity. Proc R Soc Lond B Biol Sci 2002, 269:867-872.

63. Stoehr AM, Kokko H: Sexual dimorphism in immunocompetence: what does life-history theory predict? Behav Ecol 2006, 17:751-756.

64. Zuk M: The sicker sex. PLoS Pathog 2009, 5:e1000267.

65. Clements AN: The biology of mosquitoes. Vol. 1 Development, nutrition and reproduction. Wallingford, UK: CABI Publishing; 2000.

66. Nepi M, von Aderkas P, Wagner R, Mugnaini S, Coulter A, Pacini E: Nectar and pollination drops: how different are they? Ann Botany 2009, 104:205-219.

67. Van Dyken M, Bolling BG, Moore CG, Blair CD, Beaty BJ, Black WC, Foy BD: Molecular evidence for trypanosomatids in Culex mosquitoes collected during a West Nile virus survey. Int J Parasitol 2006, 36:1015-1023.

68. Kimura M, Darbro JM, Harrington LC: Avian malaria parasites share congeneric mosquito vectors. J Parasitol 2010, 96:144-151.

69. Hargrove JW: Factors affecting density-independent survival of an island population of tsetse flies in Zimbabwe. Entomol Exp App/ 2001, 100:151-164.

70. Schwartz A, Koella JC: Melanization of Plasmodium falciparum and c-25 sephadex beads by field-caught Anopheles gambiae (Diptera: Culicidae) from southern Tanzania. J Med Entomol 2002, 39:84-88.

71. Hillyer JF, Schmidt SL, Fuchs JF, Boyle JP, Christensen BM: Age-associated mortality in immune challenged mosquitoes (Aedes aegypti) correlates with a decrease in haemocyte numbers. Cell Microbiol 2005, 7:39-51. 
72. Terzian LA, Stahler N, Irreverre F: The effects of aging, and the modifications of these effects, on the immunity of mosquitoes to malarial infection. J Immunol 1956, 76:308-313.

73. Christensen BM, Lafond MM, Christensen LA: Defense reactions of mosquitoes to filarial worms: Effect of host age on the immune response to Dirofilaria immitis microfilariae. J Parasitol 1986, 72:212-215.

74. Doums C, Moret Y, Benelli E, Schmid-Hempel P: Senescence of immune defence in Bombus workers. Ecol Entomol 2002, 27:138-144.

75. Zerofsky M, Harel E, Silverman N, Tatar M: Aging of the innate immune response in Drosophila melanogaster. Aging Cell 2005, 4:103-108.

76. Chun JD, Riehle M, Paskewitz SM: Effect of mosquito age and reproductive status on melanization of Sephadex beads in Plasmodiumrefractory and Plasmodium-susceptible strains of Anopheles gambiae. J Invertebr Pathol 1995, 66:11-17.

77. Li J, Tracy JW, Christensen BM: Relationship of hemolymph phenol oxidase and mosquito age in Aedes aegypti. J Invertebr Pathol 1992, 60:188-191.

78. Mackenzie DK, Bussiere LF, Tinsley MC: Senescence of the cellular immune response in Drosophila melanogaster. Exp Gerontol 2011, 46:853-859.

79. Whitehorn PR, Tinsley MC, Brown MJF, Darvill B, Goulson D: Genetic diversity, parasite prevalence and immunity in wild bumblebees. $\operatorname{Proc} R$ Soc B Biol Sci 2011, 278:1195-1202.

80. Moret $Y$, Schmid-Hempel P: Immune responses of bumblebee workers as a function of individual and colony age: senescence versus plastic adjustment of the immune function. Oikos 2009, 118:371-378.

81. Anderson SL, Richards SL, Tabachnick WJ, Smartt CT: Effects of West Nile virus dose and extrinsic incubation temperature on temporal progression of vector competence in Culex pipiens quinquefasciatus. J Am Mosa Control Assoc 2010, 26:103-107.

82. Krishnamoorthy K, Subramanian S, Van Oortmarssen GJ, Habbema JDF, Das PK Vector survival and parasite infection: the effect of Wuchereria bancrofti on its vector Culex quinquefasciatus. Parasitology 2004, 129:43-50.

83. Reisen WK, Meyer RP, Presser SB, Hardy JL: Effect of temperature on the transmission of western equine encephalomyelitis and Lt-Louis encephalitis viruses by Culex tarsalis (Diptera, Culicidae). J Med Entomol 1993, 30:151-160

84. Adamo SA: How should behavioural ecologists interpret measurements of immunity? Anim Behav 2004, 68:1443-1449.

85. Gonzalez-Santoyo I, Cordoba-Aguilar A: Phenoloxidase: a key component of the insect immune system. Entomol Exp App/ 2012, 142:1-16.

86. Alout H, Ndam NT, Sandeu MM, Djegbe I, Chandre F, Dabire RK, Djogbenou LS, Corbel V, Cohuet A: Insecticide resistance alleles affect vector competence of Anopheles gambiae s.s. for Plasmodium falciparum field isolates. Plos One 2013, 8:e63849.

87. Koella JC, Lynch PA, Thomas MB, Read AF: Towards evolution-proof malaria control with insecticides. Evol Appl 2009, 2:469-480

88. Read AF, Lynch PA, Thomas MB: How to make evolution-proof insecticides for malaria control. PLoS Biol 2009, 7:e1000058.

doi:10.1186/1756-3305-6-315

Cite this article as: Cornet et al: Patterns of phenoloxidase activity in insecticide resistant and susceptible mosquitoes differ between laboratory-selected and wild-caught individuals. Parasites \& Vectors 2013 6:315.

\section{Submit your next manuscript to BioMed Central and take full advantage of:}

- Convenient online submission

- Thorough peer review

- No space constraints or color figure charges

- Immediate publication on acceptance

- Inclusion in PubMed, CAS, Scopus and Google Scholar

- Research which is freely available for redistribution 